\section{RF30 BIOMARKER TESTS TO HELP DIAGNOSE PRETERM LABOUR IN SYMPTOMATIC WOMEN WITH INTACT MEMBRANES: A SYSTEMATIC REVIEW}

${ }^{1} \mathrm{M}$ Barnish*, ' J Varley-Campbell, ${ }^{1} \mathrm{H}$ Coelho, ${ }^{1} \mathrm{~S}$ Dodman, ${ }^{1,2} \mathrm{~T}$ Snowsill, ${ }^{1} \mathrm{R}$ Mújica-Mota, ${ }^{1} \mathrm{D}$ Packman, ${ }^{1} \mathrm{~N}$ Ocean, ${ }^{3} \mathrm{~T}$ Kay, ${ }^{3} \mathrm{~N}$ Liversedge, ${ }^{4} \mathrm{M}$ Parr, ${ }^{3} \mathrm{~L}$ Knight, ${ }^{1} \mathrm{C}$ Hyde, ${ }^{5} \mathrm{~A}$ Shennan, 'M Hoyle. 'Peninsular Technology Assessment Group (PenTAG), University of Exeter, Exeter, UK; ${ }^{2}$ Health Economics Group, University of Exeter, Exeter, UK; ${ }^{3}$ Obstetrics and Gynaecology, Royal Devon and Exeter NHS Foundation Trust, Exeter, UK; ${ }^{4}$ Neonatology, Central Manchester University Hospitals NHS Foundation Trust, Manchester, UK; ${ }^{5}$ Division of Women's Health, King's College London/Guy's and St Thomas' Hospital, UK

\subsection{6/jech-2018-SSMabstracts.118}

Background Preterm birth may result in short and long-term health problems for the child. Accurate diagnosis and exclusion of preterm birth could ensure appropriate admissions into hospital, transfers to specialist units and discharge home. A systematic review was conducted to assess the diagnostic accuracy of three diagnostic tests for preterm labour in symptomatic women with intact membranes.

Methods Nine bibliographic databases were searched up to July 2017 to identify studies assessing the diagnostic accuracy of fetal fibronectin ( $\mathrm{fFN}, \neq 50 \mathrm{ng} / \mathrm{ml}$ ), Actim Partus (AP) or PartoSure (PS) for predicting preterm birth within 7 days in women with intact membranes. Extensive supplementary searches were also conducted. Standardised data extraction and quality assessment using QUADAS-2 were undertaken. Narrative synthesis was used. All systematic review tasks were performed by at least 2 independent reviewers.

Results 2619 unique records were retrieved, of which 442 proceeded to full-text screening and 31 records (20 studies, $16 \mathrm{AP}, 4 \mathrm{PS}$ and $2 \mathrm{fFN}$ - some studies looked at $>1$ test) were included in the systematic review. QUADAS-2 assessment found only 5 out of 20 studies to be at low risk of bias. For AP, the best overall sensitivity and specificity results were 94.7 (95\% CI 89.9 to 97.7$)$ and 92.4 (88.9-95.1), while the worst were $33.3(4.3-77.7)$ and $74.1(69.1-78.6)$. For PS, the best overall sensitivity and specificity results were $100.0 \quad(73.5-$ $100.0)$ and 95.4 (88.6-98.7), while the worst were $0.0(0.0$ 97.5) and 97.5 (96.8-99.9). For $\mathrm{fFN}$ at $10 \mathrm{ng} / \mathrm{ml}$, sensitivity ranged from $93.8(82.8-98.7)$ to $95.7(87.8-99.1)$, and specificity from $32.2(27.7-37.0)$ to $42.3(36.5-48.4)$. For fFN at $200 \mathrm{ng} / \mathrm{ml}$, sensitivity ranged from $70.8(55.9-83.0)$ to 71.0 (58.8-81.3), and specificity from $78.6(74.3-82.5)$ to 83.6 (78.8-87.8). For fFN at $500 \mathrm{ng} / \mathrm{ml}$, sensitivity ranged from $29.2(17.0-44.1)$ to $42.0(30.2-54.5)$, and specificity from 94.3 (91.6-96.4) to 95.7 (92.7-97.8). One study compared fFN with AP and results depended on the fFN threshold used. One study compared AP and PS and found little difference in sensitivity and specificity.

Conclusion A wide range of diagnostic accuracy estimates were provided. Substantial methodological, clinical and statistical heterogeneity between studies raises considerable uncertainty about the most valid estimate of accuracy for each index test. There were few studies comparing index tests. The current results do not allow a firm conclusion as to which test is the best to use in the diagnosis of preterm labour in symptomatic women with intact membranes. Further studies comparing index tests could help to further address this question.

\section{RF31 ADVERSE CHILDHOOD EXPERIENCES OR ADVERSE SOCIO-ECONOMIC CONDITIONS? ASSESSING IMPACTS ON ADOLESCENT MENTAL HEALTH IN THE UK MILLENNIUM COHORT STUDY}

VS Straatmann*, M Whitehead, DC Taylor-Robinson. Department of Public Health and Policy, University of Liverpool, Liverpool, UK

\subsection{6/jech-2018-SSMabstracts. 119}

Background Maltreatment in childhood is associated with subsequent adverse health outcomes, but more recently the concept of 'adverse childhood experiences' (ACEs) has gaining increasing policy traction. Various adverse childhood exposures and risk conditions have been termed ACEs and have been associated with poor subsequent health outcomes, particularly mental health, but less consideration has been given to the interplay of these factors with childhood socioeconomic circumstances (SECs). We aimed to explore the social patterning of ACEs, and the extent to which ACEs explain the association of SECs and adolescent mental health.

Methods We used data from the U.K. Millennium Cohort Study based on 6748 children (9 months, 3, 5, and 14 years' old sweeps). The outcome was adolescent mental health at age 14 (Total Difficulties Score of the Strengths and Difficulties Questionnaire; $>17$ score borderline-abnormal); poverty was used as a measure of SEC at birth (Income $<60 \%$ average). ACEs were scored through 7 factors (verbal and physical maltreatment; parental drug use [age 5]; maternal domestic violence, parental divorce, maternal mental illness, and alcohol abuse [age 3 or 5]), and we generated a binary definition of exposure to 4 or more ACEs (yes/no). Counterfactual mediation analysis was used to estimate the total effect of SECs on odds of mental health problems (MHP) at age 14; and to assess the proportion mediated through exposure to multiple ACEs, using the paramed function in STATA.

Results $8.4 \%$ were exposed to multiple ACEs by age $5 \%$ and $12.6 \%$ of adolescents had MHP at age 14. Adolescent MHP and exposure to ACEs were more common among those growing up in low $v s$ high SECs, respectively (8.3\%[7.4-9.2] vs $18.8 \%[16.9-20.9]) ; 10 \%[8.2-12.1]$ vs $7.9 \%[7.0-8.9)]$. Exposure to ACEs was associated with an increased odds of MHP (OR2.2 [1.5-3.0]). The total effect of low SECs on adolescent MHP was OR $1.9(1.5-2.3)$. Overall $8 \%$ of the total effect of SEC on odds of MHP was mediated through exposure to ACEs.

Conclusion The increased risk of MHP in U.K. adolescents growing up in disadvantaged SECs is partly explained by exposure to ACEs. Children growing up in disadvantaged SECs are more at risk of experiencing multiple ACEs, and from a policy perspective it is important to separate modifiable childhood SECs from harmful exposures. Efforts to decrease inequalities in adolescents MHP should focus on reducing modifiable socio-economic inequalities; and early identification and targeted intervention for children that have experienced ACEs. The self-reported outcome is a limitation of this study. 